\title{
Analysing observer preferences when presenting a product in a rendered scene: 2D vs. autostereoscopic 3D displays
}

Francisco Felip Miralles (corresponding author)

Department of Industrial Systems Engineering and Design, Universitat Jaume I, Av. de Vicent Sos Baynat s/n, 12071 Castellón de la Plana, Spain, ffelip@uji.es Tel.: +34 964 728201; Fax: +34964728106

Elena Mulet

Department of Mechanical Engineering and Construction, Universitat Jaume I, Av. de Vicent Sos Baynat s/n, 12071 Castellón de la Plana, Spain, emulet@uji.es Tel.: +34 964 728117; Fax: +34964 728106

\section{Julia Galán Serrano}

Department of Industrial Systems Engineering and Design, Universitat Jaume I, Av. de Vicent Sos Baynat s/n, 12071 Castellón de la Plana, Spain, galan@uji.es Tel.: +34 728202; Fax: +34964728106

\section{Inmaculada Remolar Quintana}

Department of Computer Languages and Systems, Universitat Jaume I, Av. de Vicent Sos Baynat s/n, 12071 Castellón de la Plana, Spain, remolar@uji.es Tel.: +34 387687; Fax: +34964728435 


\section{Analysing observer preferences when presenting a product in a rendered scene: 2D vs. autostereoscopic 3D displays}

This research compares the way the image of a product included within a rendered scene shown on an autostereoscopic 3D display is rated versus the same image shown in a 2D display. The purpose is to understand the observer's preferences and to determine the features that a composition should have to highlight the product and to make its presentation more attractive to observers, thereby helping designers and advertisers who use both displays to prepare images to make them more effective when visually presenting a product.

The results show that observers like the images on autostereoscopic 3D displays slightly more than those presented by means of $2 \mathrm{D}$ displays. On both displays the product is perceived more quickly when it is larger than the other elements and is shown with greater chromatic contrast, but a composition is seen as more attractive when the chromatic relationship between all the elements is more harmonious.

Keywords: product presentation, observer's preference, 2D display, autostereoscopic 3D display, chromatic contrast, distance to camera.

\section{Introduction}

\subsection{Product presentation by means of an image}

Product presentation using images is currently a field of interest for design, since it allows the product to be brought closer to the consumer and to make it known in places where it would not be possible to take a physical sample, as shown by a number of studies conducted in order to understand the potential of images as a sales argument in e-retailing (Jiang and Benbasat 2007; Jeong et al. 2009; Yoo and Kim, 2014; Verhagen et al. 2014). When it comes to preparing images for the presentation of a product for a specific medium so that they will be perceived correctly and will be efficient, it is necessary to understand how the observer looks and what stimuli are perceived as more appealing. 
In this sense, two concepts emerge. On the one hand, we understand visual attention as a process in which the observer selects the most relevant information and the rest is omitted, to reduce complexity and information overload (Evans et al. 2011). On the other hand, we understand visual appeal as the quality of an image being perceived as attractive or interesting, usually at first sight (Lindgaard et al. 2006). It is difficult to determine all the factors that make an image visually pleasing, but apart from lighting conditions, scene framing and perspective (Savakis, Etz, and Loui 2000), it seems that there are two clearly related factors to visual appeal, colourfulness $(\mathrm{Cyr}$, Head, and Larios 2010) and visual complexity, where some research has shown that moderate complexity is preferred to simple or very complex stimuli (Berlyne 1971; Berlyne 1972).

Attention capturing and visual appeal are concepts related to the perception of the image that have been studied previously (Wolfe and Horowitz 2004, Gilani et al. 2013). Although it has been observed that beautiful images can attract more attention and be observed longer than less beautiful ones (Gilani et al. 2013), it has not been proven that more quickly perceived stimuli are also the most beautiful ones. In fact, many studies have investigated which parts of the image are seen earlier or which stimuli may attract more attention, as shown below.

Although it is true that on looking at a complex image its essential points can be perceived very quickly (Castelhano and Henderson 2007), it is not so easy to be sure about where an observer directs his or her gaze on seeing an image or what captures the observer's attention most. The idea that gaze is first directed towards areas of the image that are highly informative or relevant to the observer (Antes 1974) has been qualified in later studies which have shown the existence of other factors that can condition the way the observer's gaze is directed (De Graef, Christiaens, and d'Ydewalle 1990; 
Henderson, Weeks, and Hollingworth 1999). The gradual processing hypothesis posits that capturing attention is conditioned by salience, that is to say, areas of the picture in which the lighting, the contrast or the chromatic hue stand out more than in other areas, and a number of later studies have confirmed its influence (Mannen, Ruddock, and Wooding 1996; Parkhurst and Niebur 2003). Todd and Kramer (1994), however, showed that objects that are of a single colour, bright or salient but are nevertheless irrelevant to observers do not seem to capture their attention when they look at the image. Rather, in order to attract the observers' attention it is necessary for their visual field to set up a significant structure that provides novelty. In this regard, Yantis and Hillstrom (1994) showed that an increase in the luminosity of an element in an image does not necessarily have to be enough to capture the observer's attention if it does not help to transform it into a new perceptual object, or that giving the objects striking features, such as motion, does not always help to capture the observer's attention (Hillstrom and Yantis 1994).

Bearing in mind that the density of receptors in the human visual system decreases with distance from the fovea (Curcio et al. 1990), and that, therefore, visual acuity, contrast sensitivity and colour detection are lower down in the visual field periphery (DeValois and DeValois 1988; Virsu and Rovamo 1979; Newton and Eskew 2003), the stimuli of an image may go more unnoticed when they come closer to the periphery, that is, to the external limits of the image.

The location of stimuli in the left or right visual field may also influence their perception. Some studies indicate the dominance of the right hemisphere (left visual field) to process spatial information more accurately (Corballis, Funnell, and Gazzaniga, 2002; Corballis 2003), to make better size discriminations (Corballis, Funnell, and Gazzaniga, 2002) and to achieve better spatial attention (Heilman and Van Den Abell 
1979; Sturm, Reul, and Willmes 1989). In turn, some studies suggest that the reaction time to a local stimulus located in the right visual field may be shorter (Hübner 1997; Kimchi and Merhav 1991), because the left hemisphere is better than the right one for processing localised information. In any case, even though the left/right visual field may influence perception, posterior studies have shown that neuroimaging measurements do not reveal any differences in the use of brain zones (left or right) as a global property (Nielsen et al. 2013). Consequently, brain lateralisation is no longer used.

Ultimately, it is very difficult to come up with a single equation that unequivocally determines what point in the image the observer's gaze will focus on, or what elements (s)he will find more attractive, as this depends on a number of factors and many variables come into play. However, all these studies suggest that some characteristics of an object such as contrast, position to the right or left, novelty or relative size within an image may affect how quickly it is perceived.

We must also bear in mind that most studies on the behaviour of the observer's gaze have been conducted using flat images, such as photographs printed on paper or images and videos presented on 2D screens and monitors. The question then arises as to whether the way the picture of a product is perceived differs when it is presented enhancing the three-dimensional effect by using autostereoscopic 3D displays.

The field of electronic image viewing is currently marked by the coexistence of different technologies. Although LCD 2D displays with TFT transistors are the most common, there is also a gradual increase in the use of stereoscopic and autostereoscopic 3D (multi-view) displays. Whereas the first of these two systems requires the use of polarized passive glasses or active shutter glasses, the second allows the image to be observed without any kind of additional device (free-view display), which makes them 
an interesting option due to their greater comfort and allows them to be used in any setting without any kind of technical restraints.

A number of studies have evaluated the quality of the image on 3D displays and determining factors that influence the viewing experience (Ropinski et al. 2007; Jurk and Barre 2011; Hanhart, Nolfo, and Ebrahimi 2015; Duckstein et al. 2015; Kim et al. 2015; Hedberg 2013; Chen et al. 2010). Others have been conducted to determine the technical factors that can have an effect on improving the efficiency when a simulated task is performed on these devices (Sunnari et al. 2012; Sassi et al. 2014; Alpslan et al. 2005; Alpaslan et al. 2006; Yeh et al. 2008; Baer et al. 2014) or to find ways to increase the sense of naturalness perceived on observing an image using this technology (Stern and Yitzhaky 2014; Ali-Bey et al. 2013; Roger and Mårten 2010; Lewndowski et al. 2013). Despite these advances, however, today there is still a need to explore in greater depth how we see the image viewed by means of autostereoscopic displays and whether it differs from the way we see images on 2D devices.

Studies in that line would help researchers know what parts of the image are viewed first and why, which would help to identify the variables that make a picture viewed on both displays more or less attractive, revealing the keys to know how to prepare better images to present products.

\subsection{Research aim}

On the one hand, this study seeks to determine the most efficient way of presenting a product visually in a rendered scene, with a twofold aim: to determine the characteristics that an image should have in order to make the product stand out in the composition and to make the presentation image more appealing. On the other hand, it also intends to determine whether autostereoscopic 3D displays offer any advantages over conventional 2D screens when it comes to presenting a product, either because the 
image could be more attractive or because the product is perceived more quickly.

\section{Current research}

Three research questions (Q1-Q3) were formulated with the aim of determining when an element (armchair) integrated within a scene (living room) attracts the viewer's attention, considering three variables that have been considered in previous studies, as reported above. The first is the distance in relation to the camera ("y" axis); that is, an object's apparent size in relation to the rest of the image. The second variable is chromatic contrast in relation to the other elements and to the background; that is, the occurrence of differences in chromaticity in a visual scene: low, when the armchair is white, and high when it is red. The third variable is the position in relation to the horizontal axis of the composition: left or right (" $x$ " axis), in correspondence with the viewer's right and left. The last three research questions (Q4-Q6) address the question of when a composition can be found more pleasing, by taking into account the same variables.

- Q1. In a rendered scene that is viewed using displays, does the level of attention given to an element depend on its distance from the camera?

- Q2. In a rendered scene that is viewed using displays, does placing an element with high chromatic contrast with respect to its surroundings attract the viewer's attention more than if it has low chromatic contrast?

- Q3. In a rendered scene that is viewed using displays, does an element placed in the right third of the image attract the viewer's attention more than if it is situated in the left third of the picture? 
- Q4. On an autostereoscopic display, are scenes rated higher when the element to be highlighted is placed very close to the camera or when it is placed at a medium distance within the space that is being viewed?

- Q5. Are scenes rendered and viewed using an autostereoscopic display considered more pleasing when one of their elements has a colour that stands out from the others than when there is greater chromatic homogeneity among them?

- Q6. Are scenes rendered and viewed using an autostereoscopic display considered more pleasing when the main element to be highlighted is placed on the left instead of on the right?

\section{Method}

\subsection{General description}

To answer the research questions an experiment was designed consisting in generating a scene made up of the living room of a house that can be viewed on two devices: a LEDbacklit LCD 2D display and an 8-view LCD-lenticular autostereoscopic display (the technical details of which are given in section 3.3). The scene includes five commonly found items of furniture: a sofa, an armchair, a coffee table, a sideboard and a ceiling light (Figure 1). For the experiment we will take the armchair as the element that we wish to highlight. To generate variants of the same scene three variables are modified:

- The chromatic contrast of the armchair with respect to the surroundings: high (in red) and low (in white)

- The " $y$ " position of the armchair: closer to the camera (P1) or farther away and amidst the other elements (P2) 
- The "x" position of the armchair: image with the armchair on the left or mirror image (armchair on the right)

Based on these three variables, each with two levels, a total of eight rendered scene combinations are obtained (R1-R8), as summarised in Table 1. The other scene variables remain constant.

Table 1. Variants in the scene to be viewed

\begin{tabular}{cccc}
\hline Composition & $\begin{array}{c}\text { Chromatic contrast of the } \\
\text { armchair with respect to } \\
\text { the surroundings }\end{array}$ & $\begin{array}{c}\text { "y" position } \\
\text { of the armchair with } \\
\text { respect to the camera }\end{array}$ & $\begin{array}{c}\text { "x" position } \\
\text { of the armchair }\end{array}$ \\
\hline R1 & low & P2 & right \\
\hline R2 & low & P1 & left \\
\hline R3 & high & P2 & left \\
\hline R4 & hight & P1 & right \\
\hline R5 & low & P2 & left \\
\hline R6 & low & P1 & right \\
\hline R8 & high & P2 & P1 \\
\hline
\end{tabular}

\subsection{Preparation of the images (stimuli)}

Eight geometry-based 3D images (R1-R8) were created to be viewed using two types of displays. The content of the images was modelled with Autodesk 3DS Max 2016 and rendered using Mental Ray. The target camera was situated the height of the eyes of the participants, so that the perspective of the modelled room being viewed on the displays matched the observer's perspective of the real physical setting.

The room was modelled taking into account two different positions of the armchair: P1, close to the camera and P2, farther away from the camera (Figure 2) and two colour variations (red or white). Each of these four images was flipped, or mirrored, (left-right) to evaluate the possible influence of the position of the armchair (left or 
right) on the user's perception (Figure 3). The eight compositions were rendered at a resolution of 1920 x 1080 pixels. Eight views of each composition were rendered so as to be able to see the $3 \mathrm{D}$ effect on the autostereoscopic display.
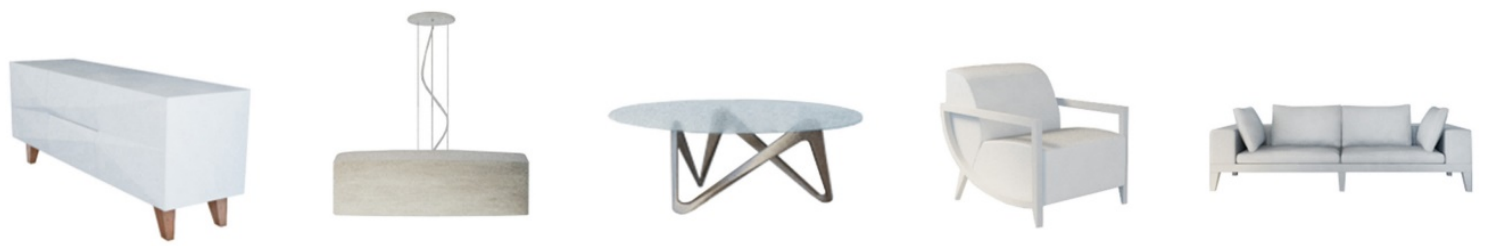

Figure 1. Items of furniture present in the living room that was viewed: sideboard, light,

coffee table, armchair and sofa
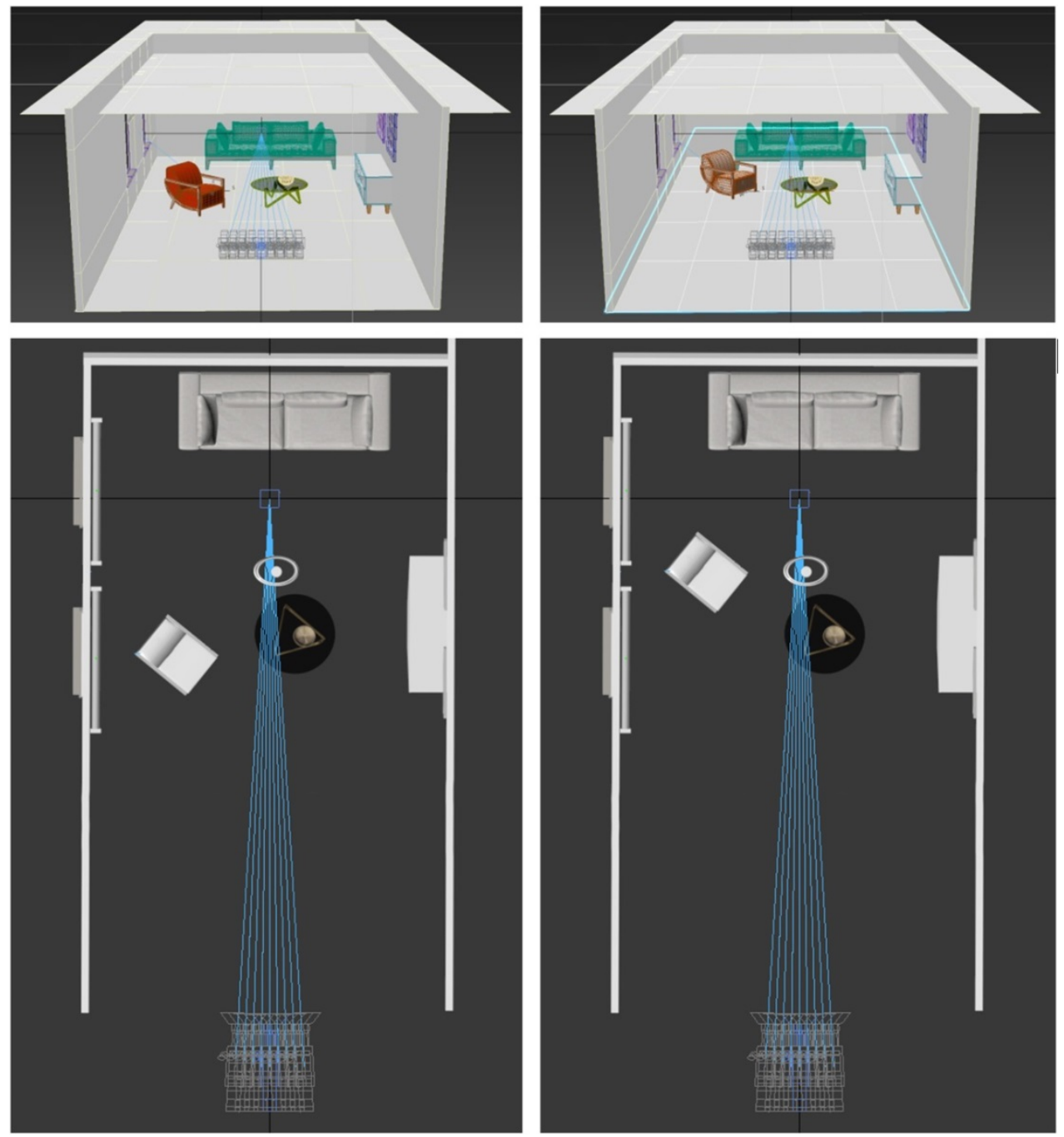

Figure 2. Aerial view of the living room that was modelled. 


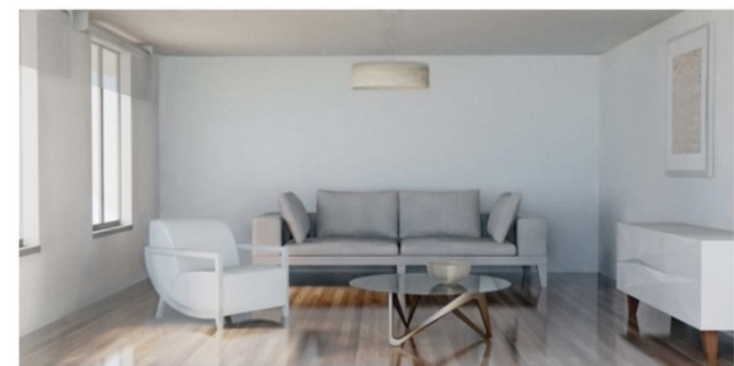

R1

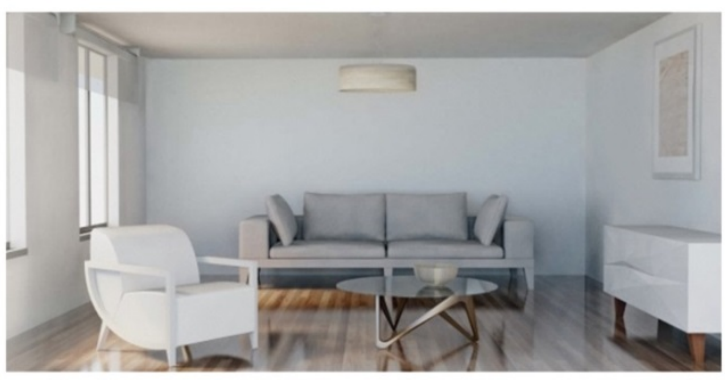

R2

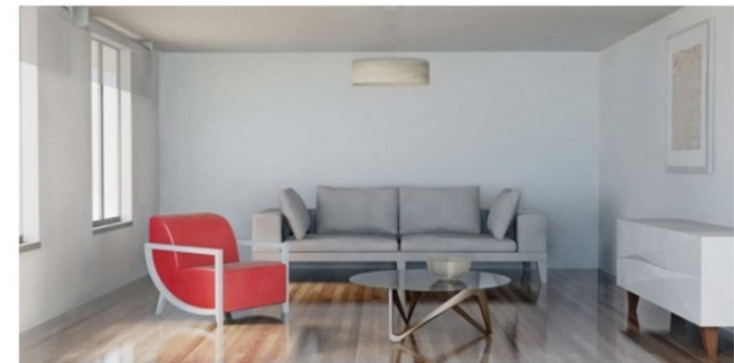

R3

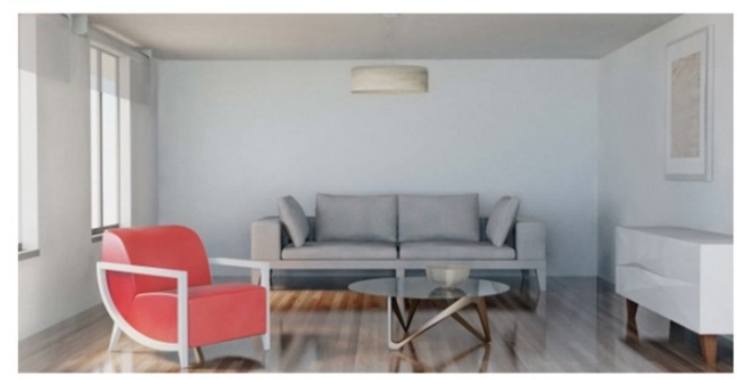

R4

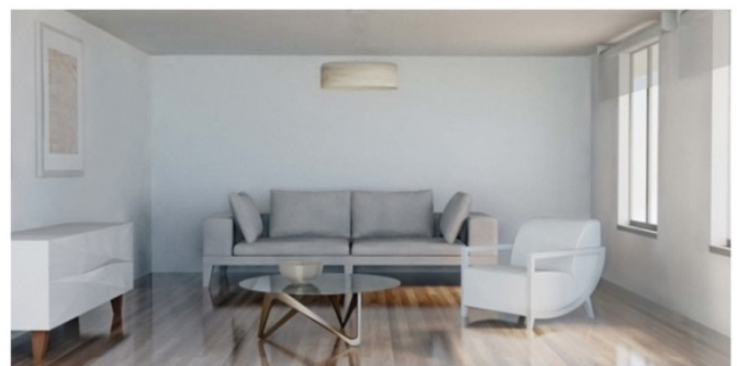

R5

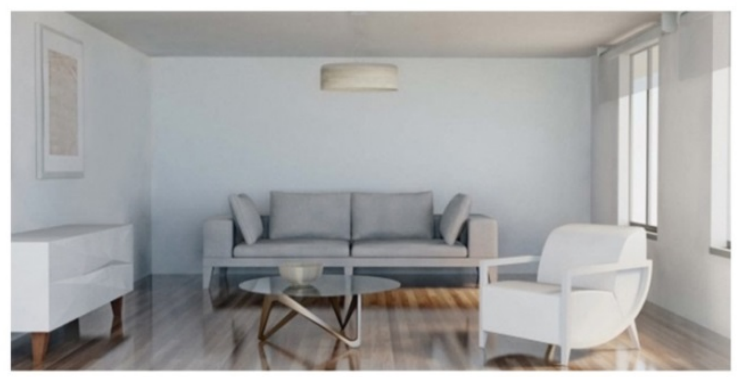

R6

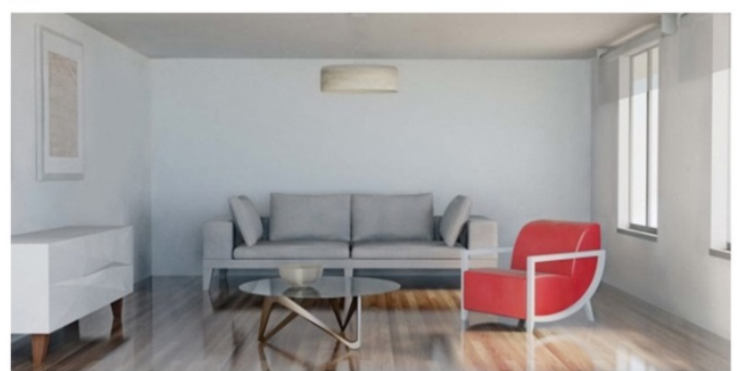

R7

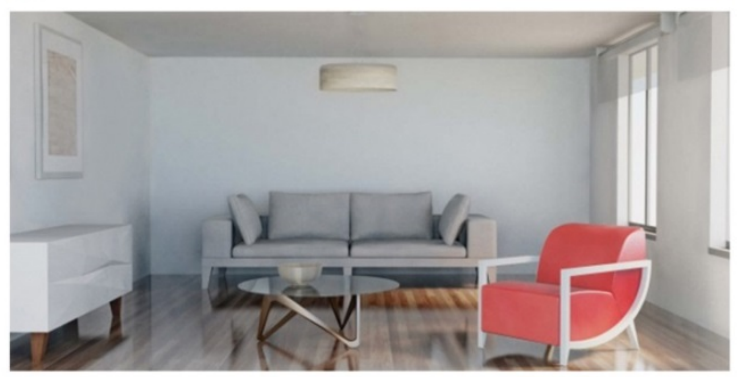

R8

Figure 3. The eight rendered compositions viewed during the experiment.

\subsection{Viewing displays}

Two displays, hereinafter denominated S-2D and S-3D, were used to view the images:

- S-2D. A 55 inches Philips 55PUH6101 6100 display, with a resolution of $3840 \mathrm{x}$ 2160 pixels (4k UHD). The technology used is LED-backlit LCD. The manufacturer does not recommend any specific viewing distance. 
- S-3D. Multi-view display model XYZ3D8V46 by Zero Creative. This is an 8view autostereoscopic display with a resolution of 1920 x 1080 pixels and a screen size of 46 inches, a brightness of $500 \mathrm{~cd} / \mathrm{m}^{2}$, a contrast ratio of 1000:1, $16.7 \mathrm{~m}$ colours and a viewing angle of approximately $100^{\circ}$. The technology used is LCD-lenticular and the viewing distance recommended by the manufacturer for an optimal 3D effect is from 3 to 7 metres.

The displays were connected to a computer which allowed a member of the research team to select the images to be viewed each time. The XYZinema 3D Video Player v1.51 software application, by Zero Creative, was used to view the images on the S-3D display, and the images were viewed on the S-2D display using Windows 7 Photo Viewer. In both cases the images were viewed full screen so as to avoid any visual interference with the graphic interfaces.

\subsection{Participants}

Altogether 92 people volunteered to view the images on the S-3D autostereoscopic display: 38 males and 54 females between 18 and 44 years old, with a mean age of 22.85 years. A total of 76 people volunteered to view the images on the S-2D display: 37 males and 39 females between 19 and 40 years old, with a mean age of 22.36 years. Most of the participants were students from the Industrial Design and Product Development Engineering degree course at the Universitat Jaume I (Spain), with no previous experience in autostereoscopic technology. Only three of them had interacted with such 3D screens before.

\subsection{Procedure}

The experiment was conducted during two sessions, separated by 5-month interval. 
During the first session, images were viewed on the S-3D display, and in the second session the S-2D display was used.

The protocol was the same for the two displays. The experiment was carried out in an empty room with no windows, but with artificial lighting. Participants were called in one by one and were asked to fill in a short questionnaire with details about aspects such as their age and gender. The questionnaire also showed the five objects depicted in Figure 1 in order to ensure that the users understood them before presenting the renders.

Then, each participant sat on an office chair, the height of which was adjusted so that their eyes were always $120 \mathrm{~cm}$ from the floor. In the case of the S-3D display, the chair was placed over a mark on the floor that was 4 metres from the screen, which was within the range of distances recommended by the manufacturer to be able to appreciate an optimal 3D effect. As the S-2D display measured 55 inches instead of 46, the chair was placed at a greater distance so that the size of the images perceived by the user was similar in both cases. Taking the two display sizes and applying the rule of three, a viewing distance of 4.78 metres was calculated for this screen (Figure. 4). 

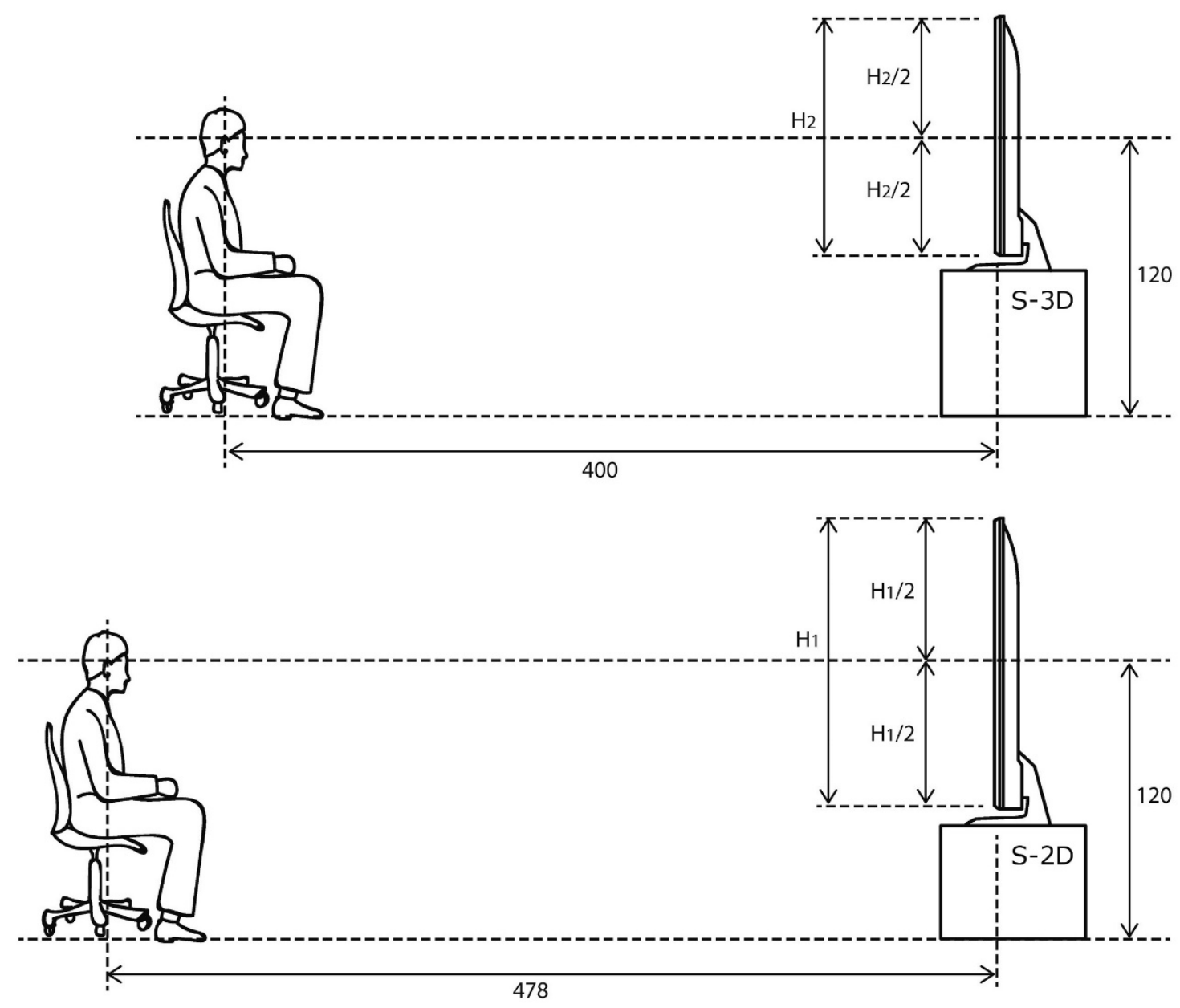

Figure 4. Diagram showing the position of the user in front of the displays.

Dimensions in $\mathrm{cm}$.

So as not to overload the participants with an excessive number of visual stimuli, each of them was shown only one group of four combinations of images: R2-R3-R5-R8 or R1-R4-R6-R7. Both these groups contain the same number of changes in the variables. To ensure that the viewing order did not affect their answers, each participant in the two groups viewed the images in random order.

Before showing the images, the lights in the room were switched off. As soon as the first scene appeared, participants had to say which element they had noticed first, that is, that which attracted their attention the most. Their oral response was recorded on a sheet of paper. The image remained on-screen for 30 seconds. Before going on to the 
next one, they were asked to give an overall score for the scene The sentence used was: "Rate on a scale of 0 to 10 points how much you like this scene globally, where 0 is the lowest value (I don't like this scene at all), 5 is the intermediate value (this scene neither pleases nor displeases me) and 10 is the highest value (I love this scene)". Their oral response was recorded. Then they were asked to close their eyes for a few seconds while the second scene was loaded on the display. This protocol was repeated until the four images had been viewed.

\section{Results and Discussion}

Figure 5 shows the ranking of the five objects according to the to the questionnaire answers. As we can see, that which the users liked the most was the coffee table, followed by the armchair and sofa. The least liked ones were the lamp and sideboard.

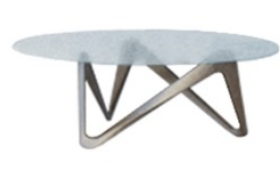

$1^{\text {st }}$

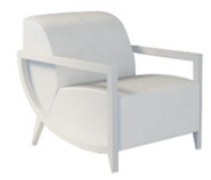

$2^{\text {nd }}$

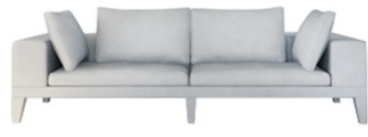

$3^{\text {rd }}$

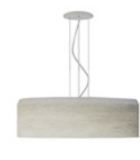

$4^{\text {th }}$

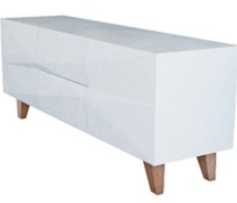

$5^{\text {th }}$

Figure 5. Ranking objects from that they liked the most to the least liked one.

Tables 2 to 4 show the number of times the first element seen by the observer was the armchair rather than one of the other items of furniture. Data were tested using the statistics software package SPSS. Since the first three research questions seek to identify whether there is a relationship between two qualitative variables, the results have to be analysed by means of the Chi-Square test $\left(\chi^{2}\right)$.

The first analysis was done to check if the number of times that an object was seen first depended on its position (closer or further away) to the camera (Q1). In Table 
2 the experimental value of the Chi-Square statistic $\left(\chi^{2} \exp \right)$ is, on both displays, greater than the theoretical value for a significance level of $\alpha=0.05$ and one degree of freedom: S-3D: Value , Cramer's V=0.281

S-2D: Value , Cramer's V=0.140

Table 2. Cross-tabulation and Chi-Square of viewing the armchair first and its distance with respect to the user

\begin{tabular}{|c|c|c|c|c|c|c|}
\hline & \multicolumn{6}{|c|}{ Displays } \\
\hline & \multicolumn{3}{|c|}{ S-3D } & \multicolumn{3}{|c|}{$\mathrm{S}-2 \mathrm{D}$} \\
\hline & $\begin{array}{l}\text { See the } \\
\text { armchair } \\
\text { first }\end{array}$ & $\begin{array}{l}\text { See another } \\
\text { object first }\end{array}$ & Total & $\begin{array}{l}\text { See the } \\
\text { armchair } \\
\text { first }\end{array}$ & $\begin{array}{l}\text { See another } \\
\text { object first }\end{array}$ & Total \\
\hline $\begin{array}{l}\text { Armchair close to the } \\
\text { camera }(\mathrm{P} 1)\end{array}$ & 132 & 52 & 184 & 112 & 40 & 152 \\
\hline $\begin{array}{l}\text { Armchair far from the } \\
\text { camera }(\mathrm{P} 2)\end{array}$ & 81 & 103 & 184 & 92 & 60 & 152 \\
\hline \multirow[t]{2}{*}{ Total } & 213 & 155 & 368 & 204 & 100 & 304 \\
\hline & & 28.992 & & & 5.961 & \\
\hline \multirow[t]{2}{*}{ V Cramer } & & 0.281 & & & 0.140 & \\
\hline & \multicolumn{6}{|c|}{3.841} \\
\hline
\end{tabular}

S: screen

Note: the values represented in italics are statistically significant ( $p$-value $<0.05)$,

Therefore the frequency with which an element was viewed first depended on its position in relation to the camera. Specifically, the armchair was viewed first more often when placed closer to the camera (P1) than when it was further away (P2). The obtained results appeared to indicate that in a rendered scene it is easier to ensure that an element is seen first by placing it closer to the camera on an autostereoscopic display than when a 2D display is used. The Cramer's V measures the correlation between the two variables. In this case, the effect of the position in relation to the camera in the frequency of times an object is first seen is small, according to the equivalences shown in Table 3. So, even though there is dependency, the effect size is small. 


\begin{tabular}{cc}
\hline Cramer's V & Effect size \\
\hline $\mathbf{0 . 1 - 0 . 3}$ & Small \\
\hline $\mathbf{0 . 3 - 0 . 5}$ & Medium \\
\hline $\mathbf{0 . 5 - 1}$ & Large \\
\hline
\end{tabular}

The next analysis was to check if the number of times that an object was seen first depended on the chromatic contrast to its surroundings (Q2). In Table 4, the experimental value also exceeds the theoretical value with both the $3 \mathrm{D}$ and the $2 \mathrm{D}$ displays:

S-3D: Value, Cramer's V= 0.369

S-2D: Value , Cramer's V $=0.350$

Table 4. Cross-tabulation of viewing the armchair first and the chromatic contrast with respect to the surroundings

\begin{tabular}{|c|c|c|c|c|c|c|}
\hline & \multicolumn{6}{|c|}{ Displays } \\
\hline & \multicolumn{3}{|c|}{$\mathrm{S}-3 \mathrm{D}$} & \multicolumn{3}{|c|}{$\mathrm{S}-2 \mathrm{D}$} \\
\hline & $\begin{array}{l}\text { See the } \\
\text { armchair } \\
\text { first }\end{array}$ & $\begin{array}{c}\text { See } \\
\text { another } \\
\text { object first }\end{array}$ & Total & $\begin{array}{l}\text { See the } \\
\text { armchair } \\
\text { first }\end{array}$ & $\begin{array}{c}\text { See } \\
\text { another } \\
\text { object first }\end{array}$ & Total \\
\hline $\begin{array}{l}\text { High chromatic contrast } \\
\text { of the armchair with } \\
\text { respect to the } \\
\text { surroundings }\end{array}$ & 140 & 44 & 184 & 127 & 25 & 152 \\
\hline $\begin{array}{l}\text { Low chromatic contrast } \\
\text { of the armchair with } \\
\text { respect to the } \\
\text { surroundings }\end{array}$ & 73 & 111 & 184 & 77 & 75 & 152 \\
\hline \multirow[t]{2}{*}{ Total } & 213 & 155 & 368 & 204 & 100 & 304 \\
\hline & & 50.036 & & & 37.255 & \\
\hline \multirow[t]{2}{*}{ V Cramer } & & 0.369 & & & 0.350 & \\
\hline & \multicolumn{6}{|c|}{3.841} \\
\hline
\end{tabular}

S: screen

Note: the values represented in italics are statistically significant ( $p$-value $<0.05$ )

Therefore, the Chi-square test showed that no variation existed in the attention paid by the observer as a result of placing an element with a high or low chromatic contrast in relation to the surroundings. When the contrast between the colour of the 
armchair and the rest of the scene was greater, more users viewed it first than when the colour of the armchair was more neutral. The Cramer's V indicated that the effect size of the chromatic contrast of an object in relation to the surroundings was medium or moderate (Table 3). So, chromatic contrast has a moderate effect to draw attention to a specific object in a scene.

The next step was to test if the number of times that an object was seen first depended on where it was placed: in the left third or the right third of the image (Q3). In Table 5 the experimental Chi-Square value is lower than the theoretical value:

S-3D: Value

S-2D: Value

The obtained results showed that the number of times that the armchair was identified as the element first seen was, in statistical terms, the same when it was placed in the left third or the right third of the image. Therefore, with the available data, it was not possible to know if the left of right position influenced the frequency of times that the object was seen first.

Table 5. Cross-tabulation of viewing the armchair first and its position in the left or right third of the image

\begin{tabular}{ccccccc}
\hline & \multicolumn{3}{c}{ Displays } \\
\cline { 2 - 7 } & $\begin{array}{c}\text { See the } \\
\text { armchair } \\
\text { first }\end{array}$ & $\begin{array}{c}\text { See } \\
\text { another } \\
\text { object first }\end{array}$ & Total & $\begin{array}{c}\text { See the } \\
\text { armchair } \\
\text { first }\end{array}$ & $\begin{array}{c}\text { See } \\
\text { another } \\
\text { object first }\end{array}$ & Total \\
\hline Armchair on the right & 107 & 77 & 184 & 105 & 47 & 152 \\
\hline Armchair on the left & 106 & 78 & 184 & 99 & 53 & 152 \\
\hline Total & 213 & 155 & 368 & 204 & 100 & 304 \\
\hline
\end{tabular}

S: screen

Note: the values represented in italics are statistically significant ( $p$-value $<0.05$ ) 
The next step consists of answering research questions Q4 to Q6, and testing whether the display type affects the scene ratings by an analysis of variance (ANOVA). Table 6 shows the mean value of the score about how much the users liked the scene. As observed, the scenes viewed on the autostereoscopic display are rated slightly better. ANOVA was applied to test whether the difference in the rating was statistically significant or not depending on the display type. The results show a difference with a significance level of $\alpha=0.05$ ( $p$-value $=0.008<0.05$ ). Therefore, rating depends on the display.

Cohen's d measures the effect size between two groups with the equivalences depicted in Table 7. The effect size of screen type is small, as Cohen's $d$ index is 0.0276 and the effect size $\mathrm{r}$ correlation is 0.1033 . So the effect is small; this is, there is a minor difference according to the display type.

Table 6. Rating of the scenes depending on the type of device

\begin{tabular}{ccc}
\hline & \multicolumn{2}{c}{ Displays } \\
\cline { 2 - 3 } & S-3D & S-2D \\
\hline Mean rating of the scene & 6.94 & 6.64 \\
\hline S.D & 1.413 & 1.476 \\
\hline 95\% confidence interval & $6.80-7.09$ & $6.47-6.81$ \\
\hline Sig. (p-value) & & 0.008 \\
\hline Cohen's d & & 0.2076 \\
\hline Coefficient $\mathbf{r}$ & & 0.1033 \\
\hline
\end{tabular}

S: screen

Note: the values represented in italics are statistically significant $(p$-value $<0.05)$

Table 7. Cohen's d index and Effect size

\begin{tabular}{cc}
\hline Cohen's d & Effect size \\
\hline 0.2 & Small \\
\hline 0.5 & Medium \\
\hline $\mathbf{0 . 8}$ & Large \\
\hline $\mathbf{1 . 3}$ & Very large \\
\hline
\end{tabular}


This result suggested that the scenes were found to be slightly more pleasing when viewed on an autostereoscopic display. Although the mean value $95 \%$ confidence interval is differed for both device types, this difference was only 0.3 on a 10 -point scale. So with collected data, it would appear that although the rating of the scene was statistically different for each device type, this difference was very small. Several factors that may have had an influence on this decision have to be taken into account. It is possible that participants who viewed the scenes with the S-3D devices gave them a higher score due to the fact that it they were viewed using a more gimmicky technology with which hardly any of them were familiar. It is also possible that the images viewed with the S-3D device were better rated because the sample consists of relatively young people, who may be more receptive to new technologies.

In addition to determining which element the participants saw first in each scene, data were also obtained concerning the score given for each of these scenes according to the study variables (Figure 6). As can be observed, the difference among these ratings becomes greater after changing the colour of the element to be highlighted (high chromatic contrast). 


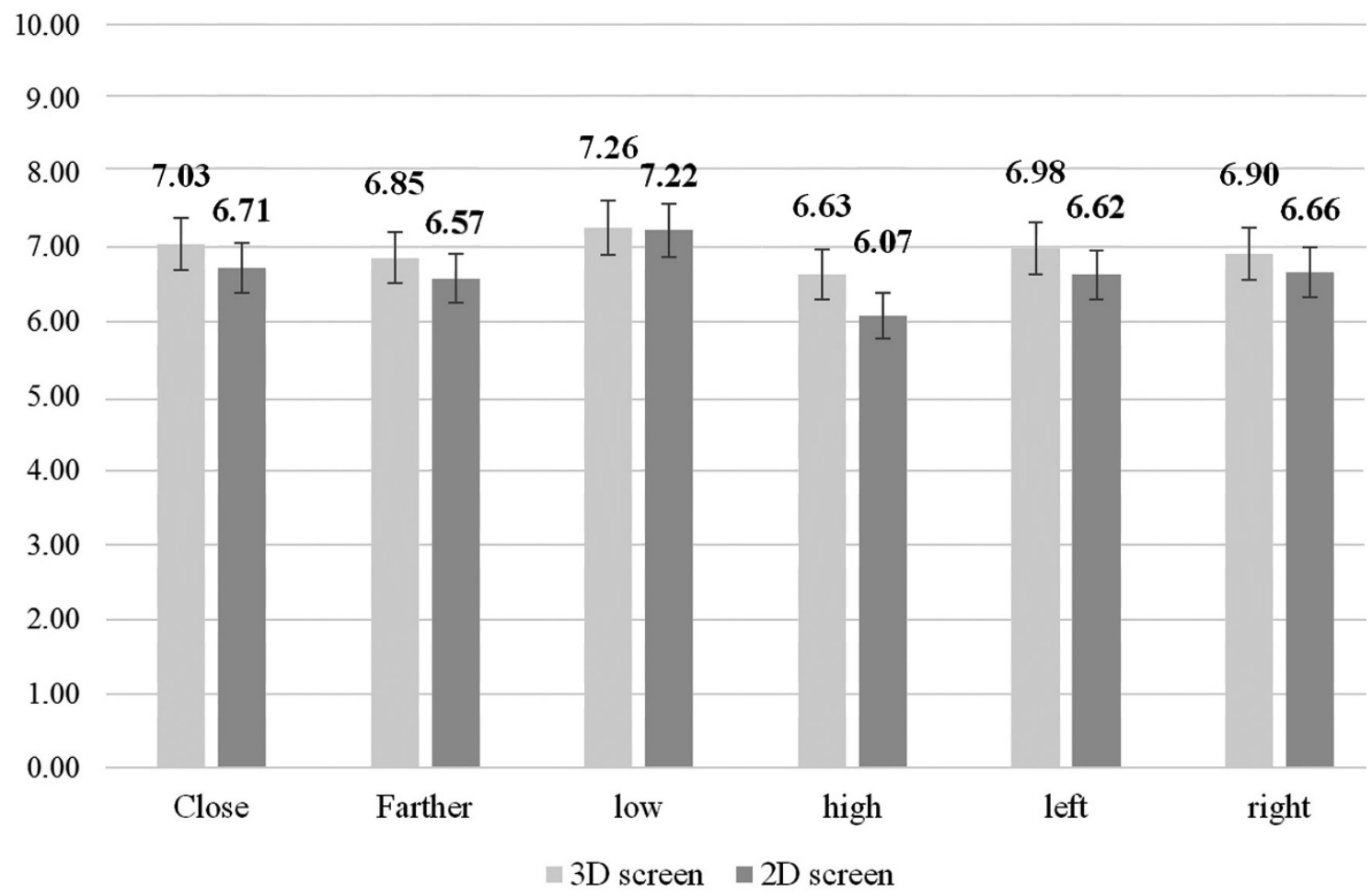

Figure 6 . The mean score ( 0 to 10$)$ given to each scene according to the variables, with error bars

To determine whether any of the modified variables have an influence on the score given to the scene viewed on the S-2D (Q4, Q5,Q6), an analysis of variance (ANOVA) was performed to test whether any of the differences in the scores are significant, taking into consideration a significance level of $\alpha=0.05$ ( $p$-value $=0.05)$ (Tables 8 and 9).

Table 8. Participants' scores on each of the scenes viewed on the S-3D

\begin{tabular}{|c|c|c|c|c|c|c|}
\hline & \multicolumn{2}{|c|}{$\begin{array}{l}\text { The " } y \text { " position of the } \\
\text { armchair with respect to } \\
\text { the camera }\end{array}$} & \multicolumn{2}{|c|}{$\begin{array}{l}\text { Chromatic contrast of the } \\
\text { armchair with respect to the } \\
\text { surroundings }\end{array}$} & \multicolumn{2}{|c|}{$\begin{array}{c}\text { The " } x \text { " position of the } \\
\text { armchair }\end{array}$} \\
\hline & Close & $\begin{array}{c}\text { Farther } \\
\text { away }\end{array}$ & Low & High & Left & Right \\
\hline Sample size & 184 & 184 & 184 & 184 & 184 & 184 \\
\hline $\begin{array}{c}\text { Mean score }(0- \\
10)\end{array}$ & 7.0326 & 6.8478 & 7.2554 & 6.625 & 6.9783 & 6.9022 \\
\hline S.D & 1.4177 & 1.40596 & 1.27394 & 1.47682 & 1.37153 & 1.45593 \\
\hline
\end{tabular}




\begin{tabular}{ccccccc}
\hline $95 \%$ & $6.8264-$ & $6.6433-$ & $7.0701-$ & $6.4102-$ & $6.7665-$ & $6.7027-$ \\
$\begin{array}{c}\text { confidence } \\
\text { interval }\end{array}$ & 7.2388 & 7.0523 & 7.4407 & 6.8398 & 7.1900 & 7.1017 \\
\hline Sig. (p-value) & & & & & \\
\hline Cohen's d & 0.21 & 0.000 & 0.606 \\
\hline
\end{tabular}

Note: the values represented in italics are statistically significant $(p$-value $<0.05)$

Table 9. Participants' scores on each of the scenes viewed on the S-2D

\begin{tabular}{|c|c|c|c|c|c|c|}
\hline & \multicolumn{2}{|c|}{$\begin{array}{l}\text { The " } y \text { " position of the } \\
\text { armchair with respect to } \\
\text { the camera }\end{array}$} & \multicolumn{2}{|c|}{$\begin{array}{l}\text { Chromatic contrast of the } \\
\text { armchair with respect to the } \\
\text { surroundings }\end{array}$} & \multicolumn{2}{|c|}{$\begin{array}{c}\text { The " } x \text { " position of the } \\
\text { armchair }\end{array}$} \\
\hline & Close & $\begin{array}{l}\text { Farther } \\
\text { away }\end{array}$ & Low & High & Left & Right \\
\hline Sample size & 152 & 152 & 152 & 152 & 152 & 152 \\
\hline $\begin{array}{c}\text { Mean score }(0- \\
10)\end{array}$ & 6.71 & 6.57 & 7.22 & 6.07 & 6.62 & 6.66 \\
\hline S.D & 1.516 & 1.436 & 1.260 & 1.454 & 1.376 & 1.574 \\
\hline $\begin{array}{l}95 \% \\
\text { confidence } \\
\text { interval }\end{array}$ & $6.47-6.95$ & $6.34-6.80$ & $7.02-7.42$ & $5.83-6.3$ & $6.40-6.84$ & $6.41-6.92$ \\
\hline Sig. (p-value) & \multicolumn{2}{|c|}{0.415} & \multicolumn{2}{|c|}{0.000} & \multicolumn{2}{|c|}{0.786} \\
\hline Cohen's d & \multicolumn{2}{|c|}{ - } & \multicolumn{2}{|c|}{0.779} & \multicolumn{2}{|c|}{ - } \\
\hline
\end{tabular}

Note: the values represented in italics are statistically significant ( $\mathrm{p}$-value $<0.05$ )

Regarding research question $4(\mathrm{Q} 4)$, the influence of how far the elements are from the camera in the rating was tested. The analysis of the variance of the results from the experiment (Tables 8 and 9) shows that with the collected data, it is not possible to state that the scenes were rated differently depending on how far elements are from the camera in the rendered environment $(p=0.21>0.05$ with the $3 \mathrm{D}$ display and $\mathrm{p}=0.415>0.05$ with the $2 \mathrm{D}$ display). That is to say, the scene was rated similarly, and regardless of whether the armchair (the element to be highlighted) was placed near the camera or further away from it. Therefore with the available data, it is not possible to assess if the position from the camera has any influence. 
One possible explanation as to why there is no marked preference for either of the two compositions used in our experiment is that they are similar: there is no big difference between the two distances separating the armchair and the camera, and the relative size of the armchair with respect to the other elements in the scene does not differ much from one scene to the other.

It must also be taken into account that an observer's preference for one type of composition or another may depend on a number of variables. Some studies claim that it is possible to understand this preference by taking into account characteristics that only concern the layout of the elements in the composition, bearing in mind factors such as symmetry, the relation between empty spaces and those occupied by objects, the number of objects close to the thirds lines on both axes, among others (Escoffery, 2012) although its study is very complex.

Regarding research question 5 (Q5), the rating of the scenes is compared depending on the chromatic homogeneity or heterogeneity of elements. The rating given by the users for the viewed scenes was significantly higher when all the elements had a higher degree of chromatic homogeneity (Tables 8 and 9): when the armchair was white -and therefore the colour scheme of the scene was more homogeneous-scores were higher (7.26 in 3D and 7.22 in 2D) than when the armchair was red (6.63 in 3D and 6.07 in $2 \mathrm{D})$. Although the differences in scores were not very large in absolute terms, in statistical terms a marked difference was found for the more homogeneous colour scheme $(\mathrm{p}=0.000<0.05)$. Thus, chromatic homogeneity or heterogeneity influenced the rating. Cohen's index shows that the chromatic contrast had a medium effect $(0.446)$ in the S-3D displays and a large effect $(0.779)$ on the S-2D. So homogeneous colour scheme was better rated, and the influence on the rating was medium or moderate for 3D displays, but was quite important for 2D displays. This may be due to the fact that 
the autostereoscopic technology does not offer the same viewing experience as that of LED-backlit LCD. To the observer, the image viewed on the 2D screen appears to be much better defined, with each element showing more details and sharper edges, and consequently the chromatic contrast of the element to be highlighted with respect to the rest of the image is far more apparent.

The study of chromatic homogeneity or the preference for a combination of colours between a figure and the background is a complex topic in which a number of issues need to be taken into account. Such aspects include the pair preference the pair harmony and the figural preference (Schloss and Palmer 2011). Palmer, Schloss and Sammartino (2013) also point out that to be able to understand the preference for one image or another it is necessary to take into account the aesthetics of the object shape and the aesthetics of the spatial composition.

In order to minimise the impact of the influence of some of these factors in our study, the rendered scenes were created with objects with a similar aesthetic appearance that were distributed in a single composition, which could be reflected as a mirrorimage. For this study it was decided that the armchair would be highlighted in red, with hue, brightness and saturation values that contrast strongly with the background, although other colours with similar properties could have been used. Likewise, in order to achieve the effect of chromatic homogeneity between the figure and the background it was decided that a neutral colour would be used for the armchair (white), similar to that of the other items of furniture in the scene.

Finally, the difference in the rating of the scene depending on it being placed in the left third or the right third of the image was tested (Q6). The results of the experiment showed that it was not possible to state that the rating of the scene varied according to whether the main element to be highlighted was in the left third (6.98 in 
$3 \mathrm{D}$ and 6.62 in 2D) or the right third of the image (6.90 in 3D and 6.66 in 2D) $(p=0.606>0.05$ and $p=0.786>0.05)$.

One possible explanation for why both scenes are found to be equally pleasing is that mirror images were used, and therefore the main element to be highlighted, the armchair, is pointing in the same direction in both images, that is, towards the inside of the composition. Recent studies have shown that consumers have a more positive perception of pictures of products that are placed pointing towards the inside of the advertising composition (Leonhardt, Catlin, and Pirouz, 2015). Therefore, in future research it would be interesting to use compositions allowing the evaluation of two other orientations of the object to be highlighted (facing inward or outward) and to determine whether in both cases the horizontal position (left or right) of this element continues to have no influence on the overall appraisal of the composition.

\section{Conclusions}

This study shows that, although images can be perceived in more detail on a $2 \mathrm{D}$ screen than on an autostereoscopic 3D one, generally speaking images presented on autostereoscopic screens are slightly better rated than those on 2D displays. In any case, the effect size is small (Cohen's $d=0.2076)$. Thus presenting products on 3D screens barely improves users' subjective perception, and the use of such devices could have a slightly more positive influence on consumers.

Variables such as the distance of an object from the camera or its chromatic contrast, in relation to other composition elements, were seen to influence whether that object was perceived more quickly than others. In line with this, an object is viewed more often when its chromatic contrast stands out much more from the rest of the scene, when placed closer to the camera and when displayed on both 3D and 2D screens. However, the influence of position is stronger on 3D displays. The data obtained herein 
do not indicate that placing objects on the right or left of the image has an influence on how pleasing observers find the composition or on which object they see first. Likewise, the chromatic homogeneity of the different elements that make up a visual composition positively influences the overall rating of the image. Based on these results, the following recommendations can be made when presenting products on autostereoscopic 3D displays:

- If the most important aim is that one particular element in a composition stands out and draws more attention, it should be placed closer to the camera than the other objects, and its chromatic contrast should stand out more. In any case, both have a small effect size. Placing the element to be highlighted more to the right than to the left of the composition is irrelevant.

- If the most important aim is to achieve an attractive scene or composition, all the elements need to maintain a chromatically harmonious relationship. This has a large effect size on 2D screens and a moderate one on 3D screens.

- Bearing these considerations in mind, in order to achieve an attractive scene in which one element stands out, this element must be larger than the other elements, and the whole image needs to have a homogeneous colour scheme.

\section{Limitations and future research}

The results of this study offer information that can be useful for designers and publicists, but several limitations would justify further research with a broader scope in the future.

In this study the users rated the images focused on a single product category, in this case different items of furniture within a domestic setting, but it would be interesting to test whether the viewing preferences with the two types of screen are 
maintained or change on observing other scenes that present products from other categories, such as household appliances, vehicles or basic necessities. Some studies have shown that the attitudes towards products depend on hedonic and utilitarian dimensions, and can vary according to specific products (Batra and Ahtola 1991) or product categories (Crowley, Spangenberg, and Hughes 1992). It would therefore be interesting to determine which of the two types of viewing display could lead to a better rating of products when one of these two dimensions is more highly developed.

In this study the user was asked to give an overall rating of a scene in which a product was presented within its context of use, with the aim of determining what viewing technology gave rise to better appraisals of the image that was observed. But another topic that would be an interesting line to investigate would be how the type of display influences the perception of quality of the product itself by comparing the case when it appears within its natural setting of use and when it appears with an empty background.

Lastly, since the sample used was made up of relatively young people, it would be a good idea to expand the sample to other sectors of the population including older people, with the aim of determining whether the viewing preferences remain the same or are affected by this factor. This would provide valuable information about how to prepare product presentations that are more effective for each type of screen, depending on the product category and the target age bracket.

\section{Acknowledgements}

This work was supported by the Spanish Ministerio de Economía y Competitividad MINECO (grant number TIN2016-75866-C3-1-R) and Universitat Jaume I (grant number P1·1B2015-30). The authors also wish to thank the designers Fernando Ulldemolins, Felipe Perelló, María José Muñoz, Laura Gimeno, Elena Sanz, Francisco 
Cueto and Paula Bañuelos for their collaboration.

\section{References}

Ali-Bey, M., N. Manamanni, and S. Moughamir. 2013. "Quality assessment of autostereoscopic rendering depending on positioning accuracy of multi-view capture systems' components." Multidimensional Systems and Signal Processing 24 (2): 255-279. doi: 10.1007/s11045-012-0195-2

Alpaslan, Z. Y., A. A. Sawchuk, A. A. Rizzo III, and S. Yeh. 2006. "Effects of Gender, Application, Experience and Constraints on Interaction Performance Using Autostereoscopic Displays." In Stereoscopic Displays and Virtual Reality Systems XIII, edited by A. Woods, N. Dodgson, J. Merritt, M. Bolas and I. McDowall, 116-127. United States: SPIE Press. doi: 10.1117/12.643125

Alpaslan, Z. Y., S. Yeh, A. A. Rizzo III, and A. A. Sawchuk. 2005. “Quantitative comparison of interaction with shutter glasses and autostereoscopic displays." In Stereoscopic Displays and Virtual Reality Systems XII, edited by A. Woods, M. Bolas, J. Merritt, and I. McDowall, 616-625. United States: SPIE Press. doi: $10.1117 / 12.589291$

Antes, J. R. 1974. “The time course of picture viewing”. Journal of Experimental Psychology 103 (1): 62-70. doi: 10.1037/h0036799

Baer, A., A. Huebler, P. Saalfeld, D. Cunningham, and B. Preim. 2014.” A Comparative User Study of a 2D and an Autostereoscopic 3D Display for a Tympanoplastic Surgery”. In Eurographics Workshop on Visual Computing for Biology and Medicine, edited by I. Viola, K. Bühler, and T. Ropinski, 181-190. Viena: Eurographics Association. doi: 10.2312/vcbm.20141190 
Batra, R., and O. T. Ahtola. 1991. "Measuring the Hedonic and Utilitarian Sources of Consumer Attitudes.” Marketing Letters 2 (2): 159-170. doi: 10.1007/BF00436035 Berlyne, D. E. 1971. Aesthetics and Psychobiology. New York: Appleton-CenturyCrofts.

Berlyne, D. E. 1972. "Experimental aesthetics.” In New Horizons in Psychology, edited by Dodwell, P.C. Harmondsworth: Penguin Blijlevens, J., M. E. H. Creusen, and J. P. L. Schoormans. 2009. "How consumers perceive product appearance: The identification of three product appearance attributes." International Journal of Design, 3 (3): 27-35.

Castelhano, M. S., and J. M. Henderson. 2007. "Initial scene representations facilitate eye movement guidance in visual search." Journal of Experimental Psychology: Human Perception and Performance 33 (4): 753-763. doi: 10.1037/0096-1523.33.4.753

Chang, W. C., and T. Y. Wu. 2007. "Exploring types and characteristics of product forms." International Journal of Design 1 (1): 3-14.

Chen, W., J. Fournier, M. Barkowsky, and P. Le Callet. 2010. "New requirements of subjective video quality assessment methodologies for 3DTV." In Video Processing and Quality Metrics 2010 (VPQM). United States: Scottsdale.

Corballis, P. M. 2003. "Visuospatial processing and the right-hemisphere interpreter." Brain and Cognition 53 (2): 171-176. doi: 10.1016/S0278-2626(03)00103-9

Corballis, P. M., M. G. Funnell, and M. S. Gazzaniga. 2002. "Hemispheric asymmetries for simple visual judgments in the split brain." Neuropsychologia 40 (4): 401-410. doi: $10.1016 / \mathrm{S} 0028-3932(01) 00100-2$

Crowley, A.E., E. R. Spangenberg, and K. R. Hughes. 1992. "Measuring the hedonic and utilitarian dimensions of attitudes toward product categories." Marketing Letters 3 (3): 239-249. doi: 10.1007/BF00994132 
Curcio, C. A., K. R. Sloan, R. E. Kalina, and A. E Hendrickson. 1990. "Human photoreceptor topography." Journal of Comparative Neurology 292 (4): 497-523. doi: $10.1002 /$ cne. 902920402

Cyr, D., M. Head, and H. Larios. 2010. “Colour Appeal in Website Design Within and Across Cultures: A Multi-method Evaluation.” International Journal of HumanComputer Studies 68 (1-2) (2010), 1-21. doi: 10.1016/j.ijhcs.2009.08.005

De Graef, P., D. Christiaens, and G. d'Ydewalle. 1990. "Perceptual effects of scene context on object identification." Psychological Research 52: 317-329. doi:

10.1007/BF00868064

DeValois, R. L., and K. K. DeValois. 1988. Spatial vision. NY: Oxford University Press.

Duckstein, B., R. Bartmann, R. Netzbandt, S. Jurk, T. Ebner, and R. Barré. 2015. "Unified crosstalk measurement method for various distances on autostereoscopic multi-view displays." In Three-Dimensional Image Processing, Measurement (3DIPM), and Applications 2015. Proc. SPIE 9393, edited by R. Sitnik, and W. Puech.

Bellingham, WA: SPIE. doi: 10.1117/12.2083336

Escoffery, D. 2012. A Framework for Learning Photographic Composition Preferences from Gameplay Data. UC Santa Cruz: Computer Science.

http://escholarship.org/uc/item/8bc2f4tt

Evans, K. K., T. S. Horowitz, P. Howe, R. Pedersini, E. Reijnen, Y. Pinto, Y. Kuzmova, and J. M. Wolfe. 2011. "Visual attention". Wiley Interdisciplinary Reviews: Cognitive Science 2 (5): 503-514. doi: 10.1002/wcs.127

Forslund, K., M. Karlsson, and R. Söderberg. 2013. "Impacts of geometrical manufacturing quality on the visual product experience." International Journal of Design 7 (1): 69-84. 
Gilani, S. O., R. Subramanian, H. Hua, S. Winkler, and S. Yen. 2013. "Impact of image appeal on visual attention during photo triaging." In 2013 IEEE International Conference on Image Processing. Melbourne: VIC. doi: 10.1109/ICIP.2013.6738048 Gutiérrez, J., F. Jaureguizar, and N. García. 2015. “Subjective Comparison of Consumer Television Technologies for 3D Visualization.” Journal of Display Technology 11 (11): 967-974. doi: 10.1109/JDT.2015.2448758

Hanhart, P., C. di Nolfo, and T. Ebrahimi. 2015. “Active crosstalk reduction system for multiview autostereoscopic displays.” In 2015 IEEE International Conference on Multimedia and Expo (ICME). Turin: IEEE. doi: 10.1109/ICME.2015.7177519 Hedberg, C. 2013. "Analysis of 3D viewing experience via subjective evaluation methods." PhD diss., Royal Institute of Technology in Stockholm. Heilman, K. M., and T. Van Den Abell. 1979. "Right hemisphere dominance for mediating cerebral activation.” Neuropsychologia 17 (3-4): 315-321. doi: $10.1016 / 0028-3932(79) 90077-0$

Henderson, J. M., P. A. Jr. Weeks, and A. Hollingworth. 1999. "Effects of semantic consistency on eye movements during scene viewing." Journal of Experimental Psychology: Human Perception and Performance 25: 210-228. doi: 10.1037/00961523.25 .1 .210

Hillstrom, A.P., and S. Yantis. 1994. "Visual motion and attentional capture." Perception \& Psychophysics 55 (4): 399-411. doi: 10.3758/BF03205298 Hübner R. 1997. “The effect of spatial frequency on global precedence and hemispheric differences." Perception and Psychophysics 59 (2): 187-201. doi: 10.3758/BF03211888 Hung, W. K., and L. L. Chen. 2012. "Effects of novelty and its dimensions on aesthetic preference in product design." International Journal of Design 6 (2): 81-90. 
Jeong, S. W., A. M. Fiore, L. S. Niehm, and F. O. Lorentz. 2009. "The role of experiential value in Online shopping: The impacts of product presentation on consumer responses towards an apparel web site." Internet Research, 19 (1): 105-124. doi: $10.1108 / 10662240910927858$

Jiang, Z., and I. Benbasat. 2007. "Investigating the influence of the functional mechanisms of online product presentations." Information Systems Research 18 (4): 454-470. doi: 10.1287/isre.1070.0124

Jurk, S., and R. Barre. 2011. "Improvements of aid free 3D Presentation." In Electronic Displays Conference. Poing: Weka. doi: 10.13140/2.1.1012.4324

Kim, D., H. Lee, S. Kim, and K. Sohn. 2015. "Effect of parallax distribution and crosstalk on visual comfort in parallax barrier autostereoscopic display." Optical Engineering 54 (5): 053107-1 - 053107-10. doi: 10.1117/1.OE.54.5.053107

Kimchi, R., and I. Merhav. 1991. "Hemispheric processing of global form, local form, and texture." Acta Psychologica 76 (2): 133-147. doi: 10.1016/0001-6918(91)90042-X Lee, K. M. 2004. “Presence, Explicated.” Communication Theory 14 (1): 27-50. doi: 10.1111/j.1468-2885.2004.tb00302.x

Leonhardt, J.M., J. R. Catlin, and D. M. Pirouz. 2015. "Is Your Product Facing the Ad's Center? Facing Direction Affects Processing Fluency and Ad Evaluation.” Journal of Advertising 44 (4): 315-325. doi: 10.1080/00913367.2015.1048911

Lewandowski, F., M. Paluszkiewicz, T. Grajek, and K. Wegner. 2013. "Methodology for 3D Video Subjective Quality Evaluation.” International Journal of Electronics and Telecommunications 59 (1): 25-32. doi: 10.2478/eletel-2013-0003

Lindgaard, G., G. Fernandes, C. Dudek, and J. Brown. 2006. “Attention web designers: You have 50 milliseconds to make a good first impression!” Behaviour and Information Technology 25 (2): 115-126. doi: 10.1080/01449290500330448 
Mannen, S. K., K. H. Ruddock, and D. S. Wooding. 1996. “The relationship between the locations of spatial features and those of fixation made during visual examination of briefly presented images." Spatial Vision 10 (3): 165-188. doi:

$10.1163 / 156856896 \times 00123$

Newton, J. R., and R. T. Jr Eskew. 2003. "Chromatic detection and discrimination in the periphery: A postreceptoral loss of color sensitivity." Visual Neuroscience 20 (5): 511521. doi: 10.1017/S0952523803205058

Nielsen, J.A., B.A. Zielinski, M.A. Ferguson, J.E. Lainhart and J.S. Anderson. 2013. "An evaluation of the left-brain vs. right-brain hypothesis with resting state functional connectivity magnetic resonance imaging". PLoS One 8 (8). doi: 10.1371/journal.pone.0071275

Palmer, S.E., K. B. Schloss, and J. Sammartino. 2013. "Visual Aesthetics and Human Preference." Annual Review of Psychology 64 (1): 77-107. doi: 10.1146/annurev-psych$120710-100504$

Parkhurst, D. J., and E. Niebur. 2003. "Scene content selected by active vision.” Spatial Vision 16 (2): 125-154. doi: 10.1163/15685680360511645

Roger, O., and S. Mårten. 2010. "Multiview image coding scheme transformations: artifact characteristics and effects on perceived 3D quality." In Proc SPIE. 7524, Stereoscopic Displays and Applications XXI, 75240Z, edited by A.Woods, N. Holliman, and N. Dodgson, 241-250. United States: SPIE Press. doi: 10.1117/12.838975 Ropinski, T., F. Steinicke, G. Bruder, and K. Hinrichs. 2007. "Focus+Content Resolution Adaption for Autostereoscopic Displays." In Smart Graphics, 7th International Symposium, edited by A. Butz, B. Fisher, A. Krüger, P. Olivier, and S. Owada, 188-193. Berlin: Heidelberg. doi: 10.1007/978-3-540-73214-3_18 
Sassi, A., P. Pöyhönen, S. Jakonen, S. Suomi, T. Capin, and J. Häkkinen. 2014.

"Enhanced user performance in an image gallery application with a mobile autostereoscopic touch display." Displays 35 (3): 152-158. doi:

10.1016/j.displa.2014.05.003

Savakis, A. E., S. P. Etz, and A. C. P. Loui. 2000. "Evaluation of image appeal in consumer photography." In Proc. SPIE Human Vision and Electronic Imaging, 111120. doi: $10.1117 / 12.387147$

Schloss, K. B., and S. E. Palmer. 2011. “Aesthetic response to color combinations: preference, harmony, and similarity." Attention, Perception \& Psychophysics 73 (2). doi: /10.3758/s13414-010-0027-0

Spreer, P., and K. Kallweit. 2014. "Augmented Reality in Retail: Assessing the Acceptance and Potential for Multimedia Product Presentation at the PoS." SOP Transactions on Marketing Research 1 (1): 23-31. doi: 10.15764/MR.2014.01002 Stern, A., Y. Yitzhaky, and B. Javidi. 2014. "Perceivable Light Fields: Matching the Requirements Between the Human Visual System and Autostereoscopic 3-D Displays.” In Proceedings of the IEEE, 1571-1587. doi: 10.1109/JPROC.2014.2348938 Steuer, J. 1992. "Defining Virtual Reality: Dimensions Determining Telepresence.” Journal of Communication 42 (4): 73-93. doi: 10.1111/j.1460-2466.1992.tb00812.x Sturm, W., J. Reul, and K. Willmes. 1989. "Is there a generalised right hemisphere dominance for mediating cerebral activation? Evidence from a choice reaction experiment with lateralized simple warning stimuli." Neuropsychologia 27 (5): 747751. doi: 10.1016/0028-3932(89)90121-8

Sunnari, M., L. Arhippainen, M. Pakanen and S. Hickey. 2012. “Studying user experiences of autostereoscopic 3D menu on touch screen mobile device." In OZCHI Proceedings of the 24th Australian Computer-Human Interaction Conference, edited by 
V. Farrell, G. Farrell, C. Chua, W. Huang, R. Vasa, and C. Woodward, 558-561. New York: ACM. doi: 10.1145/2414536.2414622

Todd, S., and A. F. Kramer. 1994. "Attentional misguidance in visual search.” Perception \& Psychophysics 56 (2): 198-210. doi: 10.3758/BF03213898

Verhagen, T., C. Vonkeman, F. Feldberg, and P. Verhagen. 2014. "Present it like it is here: creating local presence to improve online product experiences." Computers in Human Behavior 39 (C): 270-280. doi: 10.1016/j.chb.2014.07.036

Virsu, V., and J. Rovamo. 1979. "Visual resolution, contrast sensitivity, and the cortical magnification factor." Experimental Brain Research 37 (3): 475-494. doi: 10.1007/BF00236818

Wolfe, J. M., and T. S. Horowitz. 2004. "What attributes guide the deployment of visual attention and how do they do it?" Nature Reviews Neuroscience 5 (6): 495-501. doi.org/10.1038/nrn1411

Yantis, S., and A. P. Hillstrom. 1994. "Stimulus-Driven attention Capture: Evidence From Equiluminant Visual Objects." Journal of Experimental Psychology: Human Perception \& Performance 20 (1): 95-107. doi: 10.1037//0096-1523.20.1.95

Yeh, S., B. Lange, C. Y. Chang, C. Wang, A. A. Sawchuk, and A. A. Rizzo III. 2008. "Effects of stereoscopic displays and interaction devices on human motor behaviour." In The Engineering Reality of Virtual Reality 2008 (05-1-10), edited by I. McDowall, and M. Dolinsky. United States: SPIE Press. doi: 10.1117/12.767859

Yoo, J., and M. Kim. 2014. "The effects of online product presentation on consumer responses: A mental imagery perspective.” Journal of Business Research 67 (11): 24642472. doi: 10.1016/j.jbusres.2014.03.006 